\title{
Determinación de las curvas de fragilidad analítica mediante el análisis incremental dinámico en el centro de salud de Conchopata en Ayacucho,2019
}

\author{
Heber Jorge Valenzuela \\ hejova2014@ hotmail.com \\ 0000-0003-4971-0751 \\ Facultad De Ingeniería Geologíca, Minera, Metalurgia Y Geográfica \\ Universidad Nacional Mayor De San Marcos
}

\section{RESUMEN}

La presente investigación tuvo como por objetivo determinar la reducción de vulnerabilidad sísmica enfocada a edificaciones desde un punto de vista geotécnico, en la actualidad se ponen de manifestó diversos sismos que como consecuencia generan pérdidas humanas y su vez económicas. La Región de Ayacucho no es ajeno a este fenómeno es por ello que se desarrolló una metodología que permitió determinar las Curvas de Fragilidad Analítica desde un enfoque probabilístico o estadístico numérico. El Centro de Salud de Conchopata es una estructura esencial y altamente vulnerable, donde cuya función no debería interrumpirse después de que ocurra un sismo severo en el análisis probabilístico con enfoque geotécnico se obtuvo niveles de daños más probables de la estructura, en la metodología probabilística se aplicó el Método Estadístico Numérico de Simulación de Montecarlo y para el Análisis Sísmico Dinámico de la estructura el Análisis de Tiempo Historia y para el Análisis del Modelado Estructural el Software ETABS (Análisis Tridimensional Extendido de Edificaciones) y programado en el sistema de cómputo numérico Matlab (MATrix LABoratory), con lo que se generó resultados aleatorios más confiables, resultados que proporciona niveles de daño de la estructura representadas por Curvas de Fragilidad Analítica.

Palabras clave: sismo; análisis incremental dinámico; curvas de fragilidad. 


\title{
Determination of analytical brittleness curves using the dynamic incremental analysis in the health center of Conchopata in Ayacucho, 2019
}

\begin{abstract}
The objective of this research was to determine the reduction of seismic vulnerability focused on buildings from a geotechnical point of view, at present various earthquakes are manifested that as a consequence generate human and economic losses. The Ayacucho Region is not alien to this phenomenon, which is why a methodology was developed that made it possible to determine the Analytical Fragility Curves from a probabilistic or statistical numerical approach. The Conchopata Health Center is an essential and highly vulnerable structure, where its function should not be interrupted after a severe earthquake occurs. In the probabilistic analysis with a geotechnical approach, more probable damage levels of the structure were obtained, in the probabilistic methodology applied the Numerical Statistical Method of Montecarlo Simulation and for the Dynamic Seismic Analysis of the structure the Time History Analysis and for the Analysis of the Structural Modeling the ETABS Software (Extended Three-dimensional Analysis of Buildings) and programmed in the Matlab numerical computing system ( MATrix LABoratory), with which more reliable random results were generated, results that provide levels of damage to the structure represented by Analytical Fragility Curves.
\end{abstract}

Keywords: earthquake; dynamic incremental analysis; fragility curves.

Artículo recibido: 02 noviembre. 2021 Aceptado para publicación: 28 noviembre 2021 Correspondencia: hejova2014@ hotmail.com Conflictos de Interés: Ninguna que declarar 


\section{INTRODUCCIÓN}

Los criterios de Análisis Sismorresistente enfocados a Edificaciones Esenciales y con ocurrencia de sismos desde el punto de vista geotécnico están evolucionando en el mundo no sólo por la información generada por los investigadores, sino que también por la información dejada por los sismos. Estas estructuras con un comportamiento sísmico inadecuado son la principal causa de pérdidas humanas durante la ocurrencia de fuertes vibraciones, provocando pérdidas económicas inimaginables. Uno de los mecanismos que se debe aplicar de manera consecuente a fin de controlar y hacer frente a un comportamiento sísmico inadecuado es modelar los diseños estructurales de la edificación bajo el enfoque suelo estructura y tomando como referencia el estudio geotécnico realizados en la base de la estructura, la calidad de información generada en el estudio de sitio dependerá de los parámetros físico mecánicos obtenidos en el estudio geológico y geofísico, esto es una de las principales para generar valores inexactos de daño en una evaluación de riesgo sísmico conducido a grandes pérdidas humanas y por tanto económicas.

\section{MÉTODOS}

\subsection{Arquitectura}

En el predimensionamiento se tomó en cuenta la distribución uno por niveles y dos por especialidades se buscó la interrelación directa entre la arquitectura y la estructura sin dejar de lado la optimización de elementos estructurales y la correcta transmisión de cargas que es necesario llevar a cabo en estructuras hiperestáticas antes de poder calcular con precisión los esfuerzos sobre las mismas. Con el predimensionamiento se establecen las dimensiones orientativas de las secciones transversales de vigas y columnas que sirven de base para un cálculo de comprobación y reajuste de las dimensiones definitivas de las secciones.

\subsection{Estructuración}

Figura 1: Modelo 3D del Edificio en el Programa ETABS

Fuente: Elaboración propia

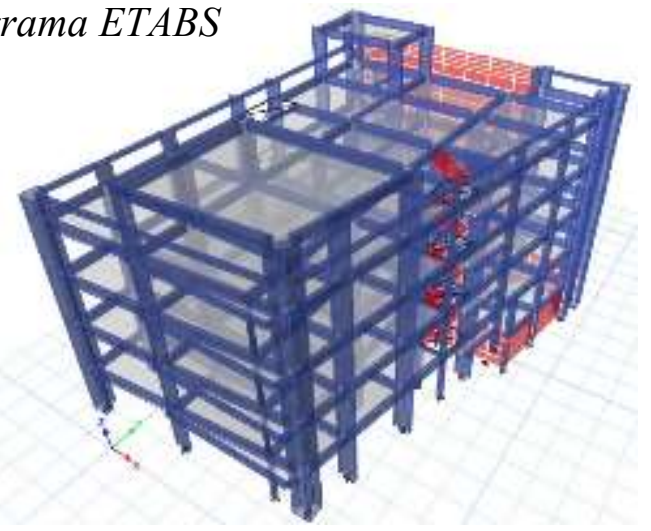


La estructura del Edificio es un Sistema dual de concreto armado constituidos por muros estructurales y pórticos, con una resistencia característica por la isometría de la forma de la estructura en el subsuelo que le corresponde únicamente al subsotano. En los techos se plantean diafragmas rígidos de losas macizas de concreto armado que descansan sobre pórticos y muros estructurales de concreto armado en una de las direcciones, con núcleos de escaleras y proyección de ascensor que se da inicio en el subsótano y termina en la azotea. Dadas las características arquitectónicas del proyecto, el tipo de suelo y la sismicidad de la zona, fue necesario incluir muros estructurales de corte en proporción importante.

\subsection{Análisis Tiempo Historia}

Se carga los datos del registro sísmico que está en formato de texto (tiempo vs aceleración), sabiendo previamente cuantos puntos contiene y la variación en el tiempo, por ejemplo, para el sismo de Pisco del 2007 se cuentan con 20,000ptos T=0.01s.

Figura 2: Archivo de acelerograma Pisco 2007

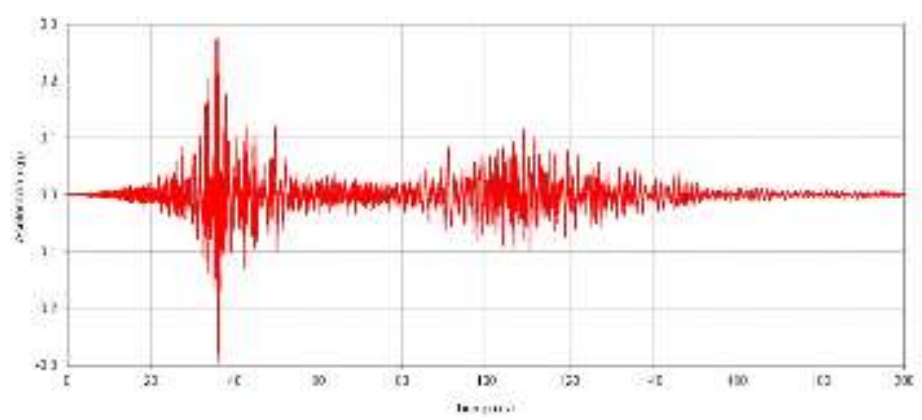

Fuente: Elaboración propia

El objetivo de un análisis Tiempo - Historia es obtener la respuesta para todo el tiempo que dura el evento, incluyendo obviamente el momento de aplicación de la máxima aceleración ósea donde podamos encontrar la respuesta de desplazamiento máximo en el pico más elevado del sismo, y continuando posteriormente durante la etapa no forzada (vibración libre).

\subsection{Deriva de piso Según Ghobarah}

Con las derivas de piso calculadas a través de los desplazamientos, resultados obtenidos del análisis dinámico tiempo historia, se procedió a clasificar las derivas de piso según los niveles de daño propuesto por (Ghobarah, 1997), permitiéndonos Ghobarah clasificar en cinco niveles de daño representado según el cuadro1, donde Ghobarah propone una metodología para clasificar el daño estructural de una edificación de concreto armado de 
tres niveles en función de la distorsión de piso obtenido del análisis dinámico tiempo historia.

Figura 3: Deriva de piso

$$
\gamma=\frac{q_{\text {ine }(i)}-q_{\text {ine }(i-1)}}{h_{i}}
$$

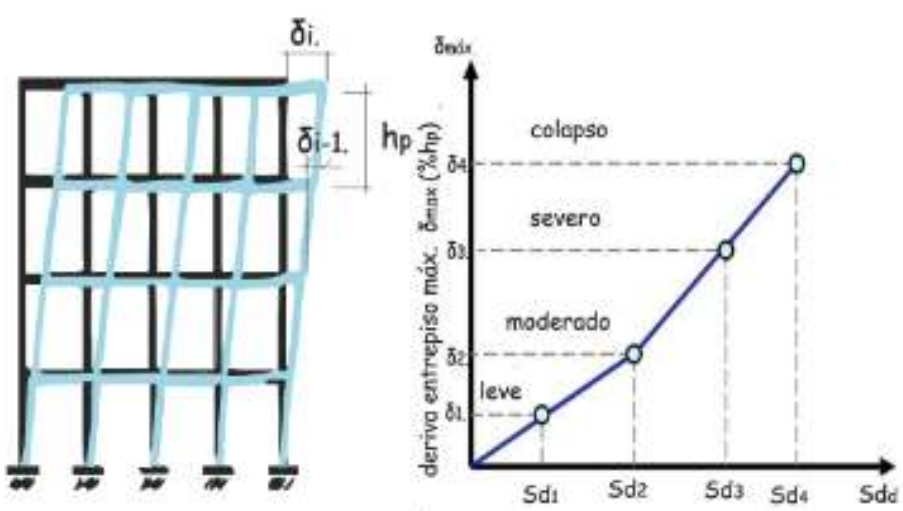

Fuente: Elaboración propia

Cuadro 1: Clasificación de la deriva de piso según Ghobarah

\begin{tabular}{|l|l|l|l|}
\hline Distorción de piso & Daño & Descripción del daño & Desempeño \\
\hline $1 Y<0.002$ & Sin daño & Sin daño & Operacional \\
\hline $0.002<Y<0.005$ & Leve & Grietas ligeramente visible & Ocupacional \\
\hline $0.005<Y<0.011$ & Moderado & Grietos menores de $1 \mathrm{~mm}$ & Seguridad de vida \\
\hline $0.011<Y<0.023$ & Extenso & Grietos entre $1 \mathrm{~mm}$ y $2 \mathrm{~mm}$ & Prevención de colapso \\
\hline$Y \geq 0.023$ & Completo & Grietos mayores a $2 \mathrm{~mm}$ & Colapso \\
\hline
\end{tabular}

Fuente: Criterios de Evaluación de desempeño estructural (Ghobarah, 1997)

\subsection{Metodología HAZUS (FEMA-NIBS, 2009)}

La Agencia Federal para el Manejo de Emergencias (FEMA) y el Instituto Nacional de Ciencias de la Construcción (NIBS), presentan una metodología para la estimación de las pérdidas por sismos en el modelo HAZUS, información muy práctica que puede ser utilizada en la evaluación de la vulnerabilidad sísmica de una ciudad o una estructura en particular en forma bastante rápida.

El modelo HAZUS provista por (FEMA \& NIBS, 2009), herramienta de análisis de peligros naturales basada en un sistema de información geográfica desarrollada y 
distribuida libremente por la Agencia Federal para el Manejo de Emergencias (FEMA), metodología que se basa en ensayos experimentales y en la opinión de los expertos en donde combina las ciencias, ingeniería y matemática, se tiene que las curvas de fragilidad, en efecto son presentadas para cuatro niveles de diseño denominados: prenorma, bajo, moderado y alto. Para cada uno de estos

niveles de diseño se tiene un límite de la deriva máxima de piso que caracteriza el nivel de daño en una estructura de concreto armado, los que se indican en los cuadros siguientes, para cuatro niveles de daño denominados: leve, moderado, extenso y completo.

Cuadro 3: Derivas de piso para estructuras de concreto armado de 1 a 3 pisos

\begin{tabular}{|l|l|l|l|l|}
\hline Niveles de daño & \multicolumn{5}{|c|}{ Niveles de daño } \\
\hline & Pre-norma & Bajo & Moderado & Alto \\
\hline Leve & 0.0040 & 0.0050 & 0.0050 & 0.0050 \\
\hline Moderado & 0.0064 & 0.0080 & 0.0087 & 0.0100 \\
\hline Extensivo & 0.0160 & 0.0020 & 0.0233 & 0.0300 \\
\hline Completo & 0.0400 & 0.0500 & 0.0600 & 0.0800 \\
\hline
\end{tabular}

Fuente: Índices de daño propuesto por HAZUS (FEMA \& NIBS, 2009)

Cuadro 4: Derivas de piso para estructuras de concreto armado de 4 a 7 pisos.

\begin{tabular}{|l|l|l|l|l|}
\hline Niveles de daño & \multicolumn{4}{|c|}{ Niveles de daño } \\
\hline & Pre-norma & Bajo & Moderado & Alto \\
\hline Leve & 0.0027 & 0.0033 & 0.0033 & 0.0033 \\
\hline Moderado & 0.0043 & 0.0053 & 0.0058 & 0.0067 \\
\hline Extensivo & 0.0107 & 0.0133 & 0.0156 & 0.0200 \\
\hline Completo & 0.0267 & 0.0333 & 0.0400 & 0.0533 \\
\hline
\end{tabular}

Fuente: Índices de daño propuesto por HAZUS (FEMA \& NIBS, 2009)

\subsection{Factores de daño medio según HAZUS (FEMA-NIBS,2009)}

Los factores de daño medio FDM o índices de daño sísmico o estado de daño en estructuras de concreto armado, se encargan de cuantificar el grado de deterioro de un elemento, grupo de elementos o de una estructura, ante la acción de un evento sísmico. 
Determinación de las curvas de fragilidad...

Cuadro 5: Factores de daño propuesto por HAZUS (FEMA \& NIBS, 2009)

\begin{tabular}{|c|c|c|c|}
\hline$N^{\circ}$ & $\begin{array}{c}\text { Grado de } \\
\text { daño }\end{array}$ & Descripción & $\begin{array}{c}\text { Perdida } \\
\text { (\%) }\end{array}$ \\
\hline 1 & $\begin{array}{c}\text { Sin daño } \\
\text { estructural }\end{array}$ & $\begin{array}{r}\text { Sin daño estructural, con posible presencia de pequeños } \\
\text { daños en componentes no estructurales }\end{array}$ & 0 \\
\hline 2 & Daño leve & $\begin{array}{c}\text { Presencia de pequeñas grietas en elementos } \\
\text { estructurales, evidente grietas en elementos no } \\
\text { estructurales, separación entre la tabiquería y los } \\
\text { elementos }\end{array}$ & 2 \\
\hline 3 & $\begin{array}{c}\text { Daño } \\
\text { moderado }\end{array}$ & $\begin{array}{r}\text { Grietas evidentes en elementos estructurales con } \\
\text { pérdida de recubrimiento en algunos casos. Grietas } \\
\text { horizontales y diagonales en tabiqueria }\end{array}$ & 10 \\
\hline 4 & Daño severo & $\begin{array}{c}\text { Falla localizada de algunos elementos estructurales. La } \\
\text { mayoría de tabiquerías presentan grandes grietas o } \\
\text { pueden volcarse, la estructura presenta una de } \\
\text { formación lateral permanente. }\end{array}$ & 50 \\
\hline 5 & $\begin{array}{r}\text { Daño } \\
\text { completo }\end{array}$ & $\begin{array}{r}\text { Desplazamiento lateral excesivo. Pérdida de la } \\
\text { estabilidad vertical, representa el colapso total o } \\
\text { parcial de la edificación. }\end{array}$ & 100 \\
\hline
\end{tabular}

Fuente: Índices de daño propuesto por HAZUS (FEMA \& NIBS, 2009)

A continuación, se muestra la figura4, puntos de los desplazamientos generados para la obtención derivas de piso que se clasificaron en la edificación según (Ghobarah, 1997), obtenidos del análisis tiempo dinámico historia sometido a 30 simulaciones de registros sísmicos, se adjunta al final $(\mathrm{E} 030,2018)$ en el anexo $\mathrm{N}$ los 30 registros sísmicos.

Figura 4: Puntos de desplazamiento para la obtención derivas de piso

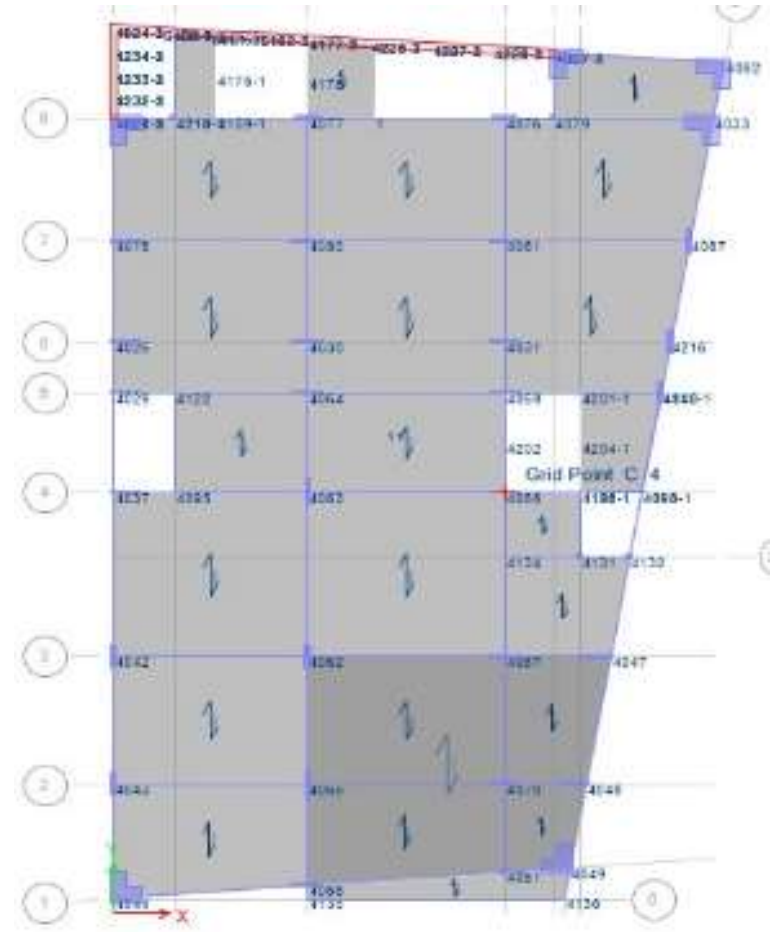

Fuente: Elaboración propia 


\section{Resultados del cálculo de Probabilidad de daño}

Cuadro 6: Probabilidad acumulada de daño leve.

\begin{tabular}{|ccc|ccc|}
\hline \multicolumn{6}{|c|}{ DAÑO LEVE } \\
\hline Nr o & Si smo & Daño Leve & Nro & Si smo & $\begin{array}{l}\text { Daño } \\
\text { Leve }\end{array}$ \\
1 & $0.16 \mathrm{~g}$ & 0.0028033 & 12 & $0.24 \mathrm{~g}$ & 0.003117 \\
2 & $0.16 \mathrm{~g}$ & 0.0045562 & 13 & $0.24 \mathrm{~g}$ & 0.004814 \\
3 & $0.16 \mathrm{~g}$ & 0.0042238 & 14 & $0.24 \mathrm{~g}$ & 0.002864 \\
4 & $0.16 \mathrm{~g}$ & 0.0024535 & 15 & $0.26 \mathrm{~g}$ & 0.003433 \\
5 & $0.18 \mathrm{~g}$ & 0.0031968 & 16 & $0.27 \mathrm{~g}$ & 0.004814 \\
6 & $0.18 \mathrm{~g}$ & 0.0048141 & 17 & $0.30 \mathrm{~g}$ & 0.003914 \\
7 & $0.18 \mathrm{~g}$ & 0.0026415 & 18 & $0.30 \mathrm{~g}$ & 0.004814 \\
8 & $0.18 \mathrm{~g}$ & 0.0031173 & 19 & $0.30 \mathrm{~g}$ & 0.002926 \\
9 & $0.19 \mathrm{~g}$ & 0.0026415 & 20 & $0.30 \mathrm{~g}$ & 0.002187 \\
10 & $0.19 \mathrm{~g}$ & 0.0035526 & 21 & $0.38 \mathrm{~g}$ & 0.002831 \\
11 & $0.19 \mathrm{~g}$ & 0.004133 & & & \\
\hline
\end{tabular}

Fuente: Elaboración propia

Cuadro 7: Probabilidad acumulada de daño moderado.

\begin{tabular}{|ccc|ccc|ccc|}
\hline \multicolumn{7}{|c|}{ DANO MODERADO } \\
\hline Nro & Si smo & $\begin{array}{c}\text { Dano } \\
\text { Mbder ado }\end{array}$ & Nro & Si smo & $\begin{array}{c}\text { Daño } \\
\text { Mbder ado }\end{array}$ & No & Si smo & $\begin{array}{c}\text { Daño } \\
\text { Moder ado }\end{array}$ \\
1 & $0.16 g$ & 0.0080690 & 19 & $0.28 g$ & 0.0060497 & 36 & $0.36 g$ & 0.0066339 \\
2 & $0.18 g$ & 0.0106917 & 20 & $0.28 g$ & 0.0064528 & 37 & $0.36 g$ & 0.0060497 \\
3 & $0.19 g$ & 0.0061886 & 21 & $0.28 g$ & 0.0064813 & 38 & $0.37 g$ & 0.0064528 \\
4 & $0.19 g$ & 0.0061886 & 22 & $0.28 g$ & 0.0051288 & 39 & $0.37 g$ & 0.0063135 \\
5 & $0.19 g$ & 0.0106917 & 23 & $0.29 g$ & 0.0066339 & 40 & $0.37 g$ & 0.0063870 \\
6 & $0.19 g$ & 0.0050353 & 24 & $0.29 g$ & 0.0051288 & 41 & $0.37 g$ & 0.0064402 \\
7 & $0.23 g$ & 0.0087429 & 25 & $0.29 g$ & 0.0066339 & 42 & $0.38 g$ & 0.0064812 \\
8 & $0.23 g$ & 0.0106917 & 26 & $0.29 g$ & 0.0055620 & 43 & $0.38 g$ & 0.0051372 \\
9 & $0.23 g$ & 0.0061886 & 27 & $0.30 g$ & 0.0054673 & 44 & $0.38 g$ & 0.0093087 \\
10 & $0.23 g$ & 0.0082381 & 28 & $0.33 g$ & 0.0060497 & 45 & $0.39 g$ & 0.0067065 \\
11 & $0.24 g$ & 0.0106917 & 29 & $0.33 g$ & 0.0060497 & 46 & $0.39 g$ & 0.0061149 \\
12 & $0.24 g$ & 0.0061886 & 30 & $0.33 g$ & 0.0064813 & 47 & $0.39 g$ & 0.0059418 \\
13 & $0.26 g$ & 0.0092669 & 31 & $0.33 g$ & 0.0060497 & 48 & $0.39 g$ & 0.0091379 \\
14 & $0.26 g$ & 0.0092669 & 32 & $0.34 g$ & 0.0064528 & 49 & $0.42 g$ & 0.0051288 \\
15 & $0.26 g$ & 0.0060497 & 33 & $0.34 g$ & 0.0055620 & 50 & $0.50 g$ & 0.0066339 \\
16 & $0.27 g$ & 0.0065361 & 34 & $0.34 g$ & 0.0054673 & 51 & $0.50 g$ & 0.0064951 \\
17 & $0.27 g$ & 0.0057022 & 35 & $0.34 g$ & 0.0051288 & 52 & $0.54 g$ & 0.0067065 \\
18 & $0.27 g$ & 0.0064528 & & & & & & \\
\hline
\end{tabular}

Fuente: Elaboración propia

Cuadro 8: Probabilidad acumulada de daño extensivo.

\begin{tabular}{|ccccccccc|}
\hline \multicolumn{7}{c|}{ DANO SEVERo (EXTENSi VO) } \\
\hline Nro & Si smo & $\begin{array}{c}\text { Daño } \\
\text { Ext ensi vo }\end{array}$ & Nro & Si smo & $\begin{array}{c}\text { Daño } \\
\text { Ext ensi vo }\end{array}$ & Nro & Si smo & $\begin{array}{c}\text { Daño } \\
\text { Ext ensi vo }\end{array}$ \\
1 & $0.19 \mathrm{~g}$ & 0.0117803 & 16 & $0.36 \mathrm{~g}$ & 0.0158024 & 31 & $0.48 \mathrm{~g}$ & 0.0160556 \\
2 & $0.23 \mathrm{~g}$ & 0.0166494 & 17 & $0.37 \mathrm{~g}$ & 0.0120072 & 32 & $0.48 \mathrm{~g}$ & 0.0164519 \\
3 & $0.26 \mathrm{~g}$ & 0.0175841 & 18 & $0.37 \mathrm{~g}$ & 0.0174374 & 33 & $0.50 \mathrm{~g}$ & 0.0162307 \\
4 & $0.27 \mathrm{~g}$ & 0.0185565 & 19 & $0.37 \mathrm{~g}$ & 0.0165566 & 34 & $0.50 \mathrm{~g}$ & 0.0160492 \\
5 & $0.28 \mathrm{~g}$ & 0.0162459 & 20 & $0.37 \mathrm{~g}$ & 0.0170343 & 35 & $0.52 \mathrm{~g}$ & 0.0159425 \\
6 & $0.29 \mathrm{~g}$ & 0.0164519 & 21 & $0.37 \mathrm{~g}$ & 0.0165466 & 36 & $0.52 \mathrm{~g}$ & 0.0121838 \\
7 & $0.31 \mathrm{~g}$ & 0.0195529 & 22 & $0.38 \mathrm{~g}$ & 0.0164519 & 37 & $0.52 \mathrm{~g}$ & 0.0173042 \\
8 & $0.31 \mathrm{~g}$ & 0.0166494 & 23 & $0.39 \mathrm{~g}$ & 0.0160492 & 38 & $0.52 \mathrm{~g}$ & 0.0174374 \\
9 & $0.31 \mathrm{~g}$ & 0.0175841 & 24 & $0.41 \mathrm{~g}$ & 0.0153531 & 39 & $0.54 \mathrm{~g}$ & 0.0170343 \\
10 & $0.31 \mathrm{~g}$ & 0.0172864 & 25 & $0.41 \mathrm{~g}$ & 0.0158643 & 40 & $0.54 \mathrm{~g}$ & 0.0161225 \\
11 & $0.33 \mathrm{~g}$ & 0.0174374 & 26 & $0.41 \mathrm{~g}$ & 0.0118371 & 41 & $0.55 \mathrm{~g}$ & 0.0167556 \\
12 & $0.34 \mathrm{~g}$ & 0.0138959 & 27 & $0.42 \mathrm{~g}$ & 0.0176189 & 42 & $0.55 \mathrm{~g}$ & 0.0174374 \\
13 & $0.35 \mathrm{~g}$ & 0.0164519 & 28 & $0.42 \mathrm{~g}$ & 0.0174374 & 43 & $0.70 \mathrm{~g}$ & 0.0165466 \\
14 & $0.35 \mathrm{~g}$ & 0.0161226 & 29 & $0.42 \mathrm{~g}$ & 0.0167475 & & & \\
15 & $0.36 \mathrm{~g}$ & 0.0160492 & 30 & $0.48 \mathrm{~g}$ & 0.0170343 & & & \\
\hline
\end{tabular}

Fuente: Elaboración propia 
Determinación de las curvas de fragilidad...

Cuadro 9: Probabilidad acumulada de daño colapso.

\begin{tabular}{|c|c|c|c|c|c|c|c|c|}
\hline \multicolumn{9}{|c|}{ DANOO COMPLETO (COLAPSO) } \\
\hline No & Sismo & $\begin{array}{c}\text { Daño } \\
\text { Compl et o }\end{array}$ & No & S sino & $\begin{array}{c}\text { Daño } \\
\text { Compl st o }\end{array}$ & No & Si smo & $\begin{array}{c}\text { Daño } \\
\text { Compl et o }\end{array}$ \\
\hline 1 & 0. $19 \mathrm{~g}$ & 0.0732032 & 15 & $0.44 \mathrm{~g}$ & 0.0560393 & 29 & $0.53 \mathrm{~g}$ & 0.0568809 \\
\hline 2 & $0.31 \mathrm{~g}$ & 0.071967 & 16 & $0.47 \mathrm{~g}$ & 0.0717316 & 30 & $0.54 \mathrm{~g}$ & 0.0567565 \\
\hline 3 & $0.35 \mathrm{~g}$ & 0.0731782 & 17 & $0.47 g$ & 0.0722585 & 31 & $0.55 \mathrm{~g}$ & 0.0559311 \\
\hline 4 & $0.35 g$ & 0.0575356 & 18 & 0.479 & 0.0724488 & 32 & $0.55 \mathrm{~g}$ & 0.0558126 \\
\hline 5 & $0.35 g$ & 0.0565662 & 19 & $0.47 g$ & 0.0730099 & 33 & $0.55 \mathrm{~g}$ & 0.0716556 \\
\hline 6 & $0.36 \mathrm{~g}$ & 0.0565662 & 20 & $0.47 g$ & 0.0717316 & 34 & $0.56 \mathrm{~g}$ & 0.0715813 \\
\hline 7 & $0.37 g$ & 0.0721618 & 21 & $0.48 g$ & 0.0570717 & 35 & $0.56 \mathrm{~g}$ & 0.0716225 \\
\hline 8 & $0.41 \mathrm{~g}$ & 0.0575356 & 22 & $0.48 g$ & 0.0719670 & 36 & $0.56 \mathrm{~g}$ & 0.0568809 \\
\hline 9 & $0.41 \mathrm{~g}$ & 0.0730099 & 23 & $0.50 \mathrm{~g}$ & 0.071967 & 37 & $0.56 \mathrm{~g}$ & 0.0717316 \\
\hline 10 & $0,42 g$ & 0.072764 & 24 & $0.52 \mathrm{~g}$ & 0.056469 & 38 & $0.56 \mathrm{~g}$ & 0.0715003 \\
\hline 11 & $0,44 \mathrm{~g}$ & 0.0722585 & 25 & $0.53 \mathrm{~g}$ & 0.0722585 & 39 & $0.70 \mathrm{~g}$ & 0.0558844 \\
\hline 12 & $0.44 g$ & 0.0732032 & 26 & $0.53 \mathrm{~g}$ & 0.071967 & 40 & $0.70 \mathrm{~g}$ & 0.0714769 \\
\hline 13 & 0.449 & 0.0717316 & 27 & $0.53 g$ & 0.0730099 & 41 & $0.70 \mathrm{~g}$ & D. 0732032 \\
\hline 14 & $0.44 g$ & 0.071967 & 28 & $0.53 g$ & 0.0565662 & 42 & $0.70 \mathrm{~g}$ & 0.0568809 \\
\hline
\end{tabular}

Fuente: Elaboración propia

\subsection{Curvas de Fragilidad}

Para el proceso de generación de curvas de fragilidad se ha implementado en un programa desarrollado en MatLab 7.10.0 (R2010a), donde la primera etapa es obtener la función de distribución acumulada de la aceleración del sismo (Sag) clasificado para cada estado de daño.

Figura 5: Diagrama de flujo para programar la probabilidad acumulada de daño

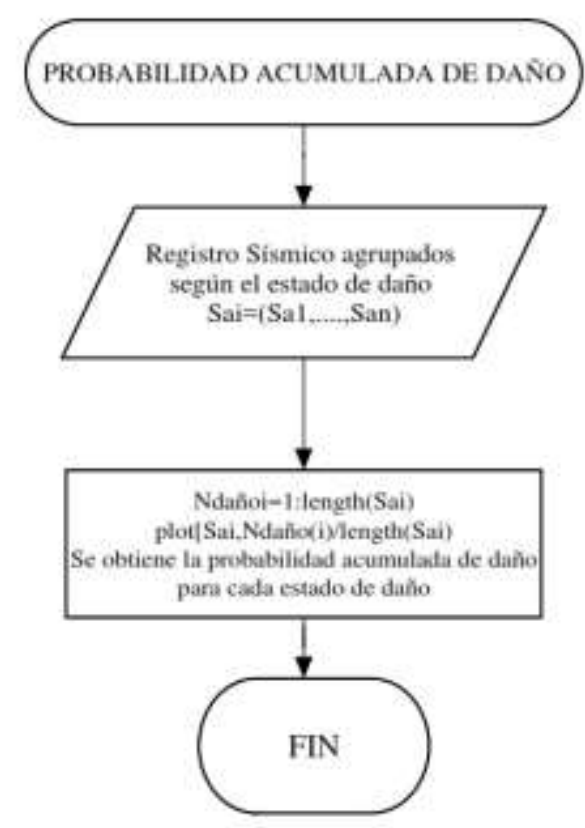

Fuente: Elaboración propia 
Ejemplo para la obtención de la curva de probabilidad acumulada de daño leve 11 según los datos de la tabla, para obtener la figura.

Cuadro 10: Probabilidad acumulada de daño leve.

\begin{tabular}{|ccc|ccc|}
\hline \multicolumn{5}{|c|}{ DANNo LEVE } \\
\hline Nro & Si smo & Daño Leve & Nro & Si smo & $\begin{array}{c}\text { Daño } \\
\text { Leve }\end{array}$ \\
1 & $0.16 \mathrm{~g}$ & 0.0028033 & 12 & $0.24 \mathrm{~g}$ & 0.003117 \\
2 & $0.16 \mathrm{~g}$ & 0.0045562 & 13 & $0.24 \mathrm{~g}$ & 0.004814 \\
3 & $0.16 \mathrm{~g}$ & 0.0042238 & 14 & $0.24 \mathrm{~g}$ & 0.002864 \\
4 & $0.16 \mathrm{~g}$ & 0.0024535 & 15 & $0.26 \mathrm{~g}$ & 0.003433 \\
5 & $0.18 \mathrm{~g}$ & 0.0031968 & 16 & $0.27 \mathrm{~g}$ & 0.004814 \\
6 & $0.18 \mathrm{~g}$ & 0.0048141 & 17 & $0.30 \mathrm{~g}$ & 0.003914 \\
7 & $0.18 \mathrm{~g}$ & 0.0026415 & 18 & $0.30 \mathrm{~g}$ & 0.004814 \\
8 & $0.18 \mathrm{~g}$ & 0.0031173 & 19 & $0.30 \mathrm{~g}$ & 0.002926 \\
9 & $0.19 \mathrm{~g}$ & 0.0026415 & 20 & $0.30 \mathrm{~g}$ & 0.002187 \\
10 & $0.19 \mathrm{~g}$ & 0.0035526 & 21 & $0.38 \mathrm{~g}$ & 0.002831 \\
11 & $0.19 \mathrm{~g}$ & 0.004133 & & & \\
\hline
\end{tabular}

Fuente: Elaboración propia

Figura 6: Probabilidad acumulada de daño leve

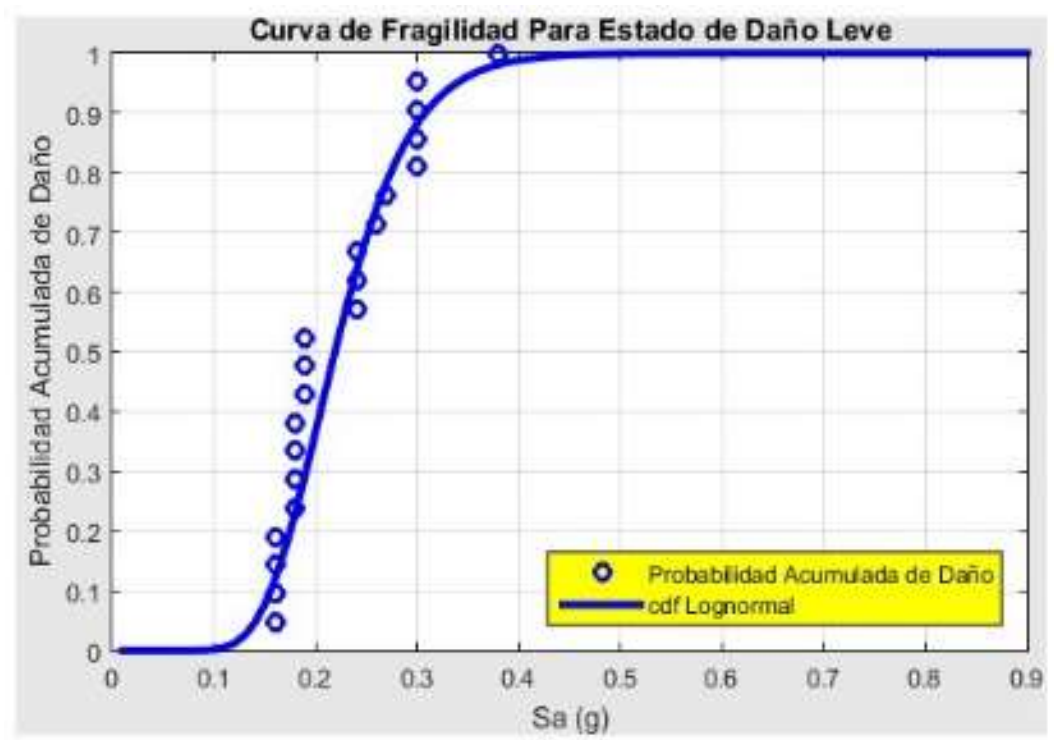

Fuente: Elaboración propia

Mediante la función de la distribución de probabilidad log normal se ajusta la gráfica anterior para todos los estados de daño: 


$$
\begin{aligned}
& P\left(C / S_{\sigma}\right)=\phi\left(\frac{\ln x-\mu}{\beta}\right) \\
& \mu-\frac{1}{n} \sum_{i=1}^{n} \ln S a_{i} \\
& \beta=\sqrt{\frac{1}{n-1} \sum_{i=1}^{n}\left(\ln S a_{i}-\hat{\mu}^{2}\right)}
\end{aligned}
$$

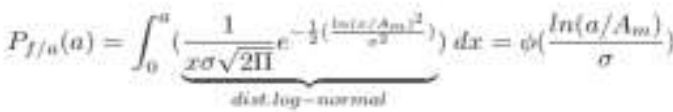

$\varnothing$ : es la función de distribución acumulativa gausiana estándar, Por lo tanto, la curva de fragilidad está totalmente definida por dos parámetros que son:

Am: Media

$\sigma:$ desviación estándar logarítmica

\section{En matlab el código es:}

media1 $=$ mean $(\log ($ leve 1$))$;

desv_estandar $1=\operatorname{std}(\log ($ leve 1$))$;

p_collapse1=logncdf(x_vals 1 , media1,desv_estandar1);

La distribución logarítmica normal es aplicable cuando la cantidad de interés debe ser positivo, ya que existe $\log (\mathrm{x})$ cuando $\mathrm{x}$ es positivo, es una distribución normal asimétrica y se usa para para modelar tiempos de procesos y reparación, averías de un coche con el tiempo, población de un sitio con respecto al dinero, estancia de tiempo en un banco, etc. En excel:

=DISTR.LOG.NORM(C2, media, desviación)

Figura 7: Diagrama de flujo para la función de dist. log normal

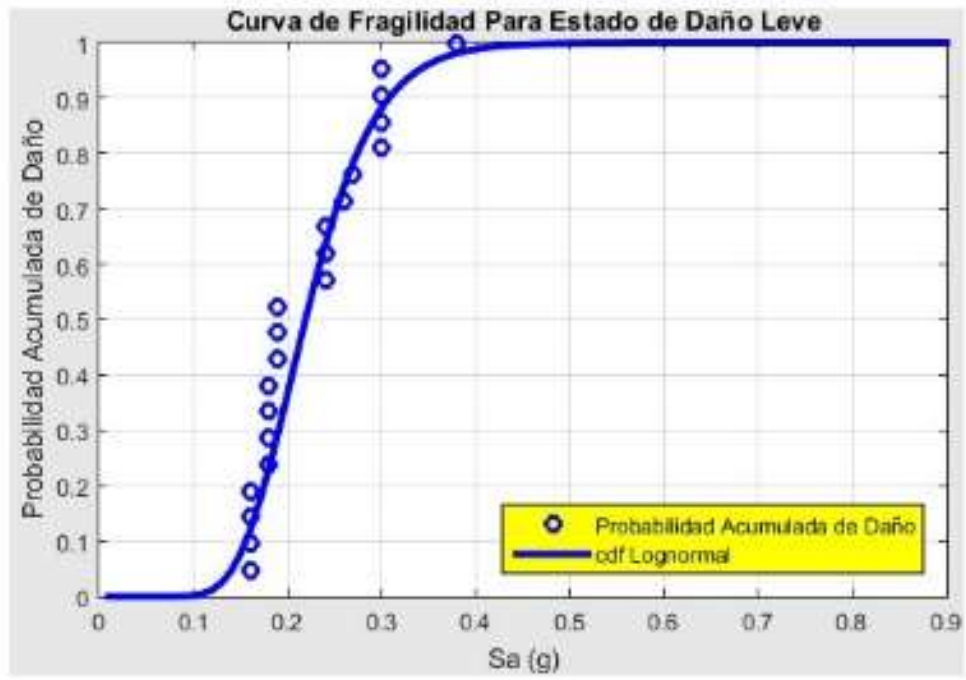

Fuente: Elaboración propia 
Figura 8: Probabilidad acumulada de daño leve y función de distribución log normal.

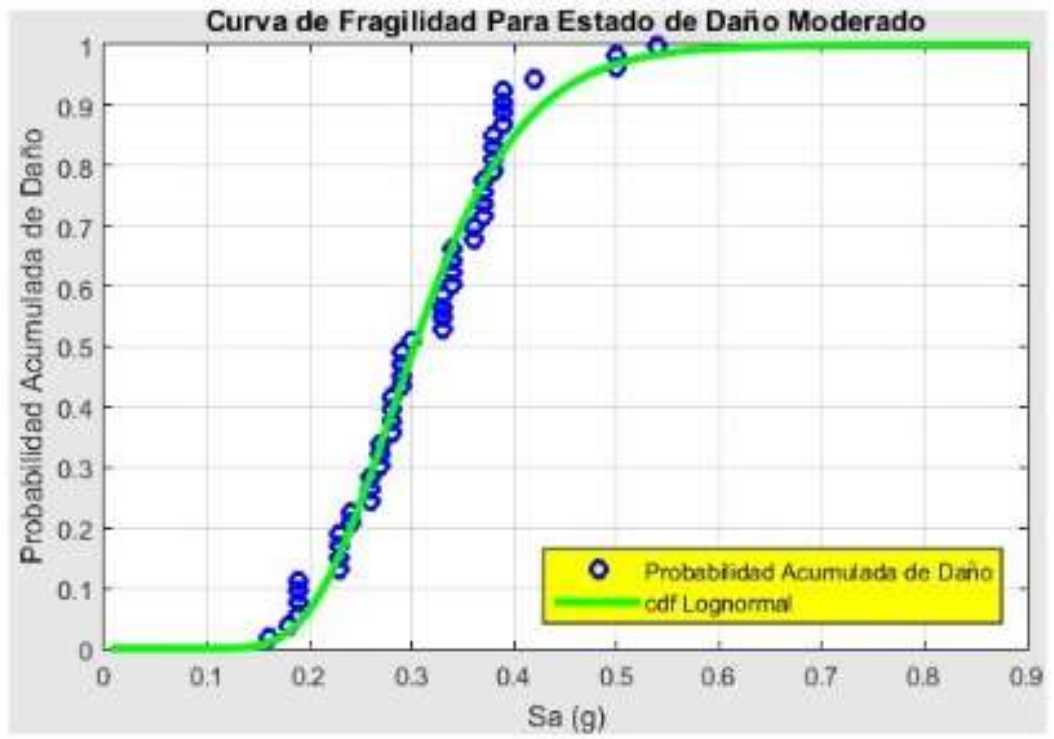

Fuente: Elaboración propia

Figura 9: Probabilidad acumulada de daño moderado y función de distribución log normal.

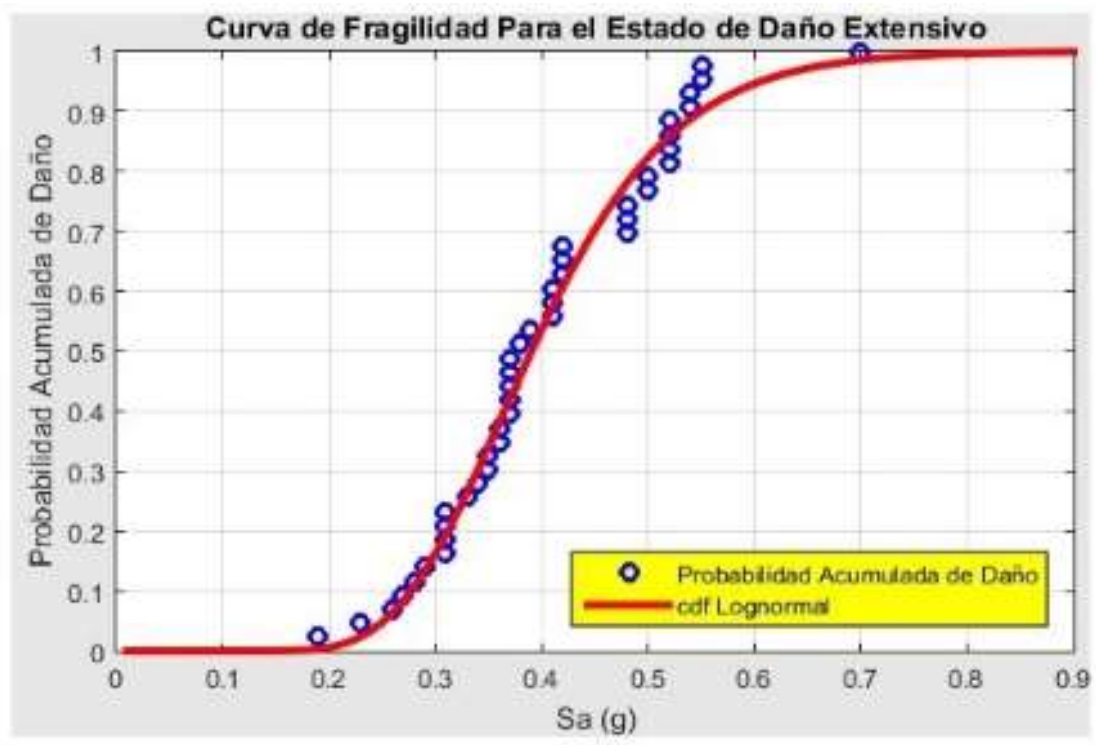

Fuente: Elaboración propia 
Figura 10: Probabilidad acumulada de daño severo y función de distribución log normal.

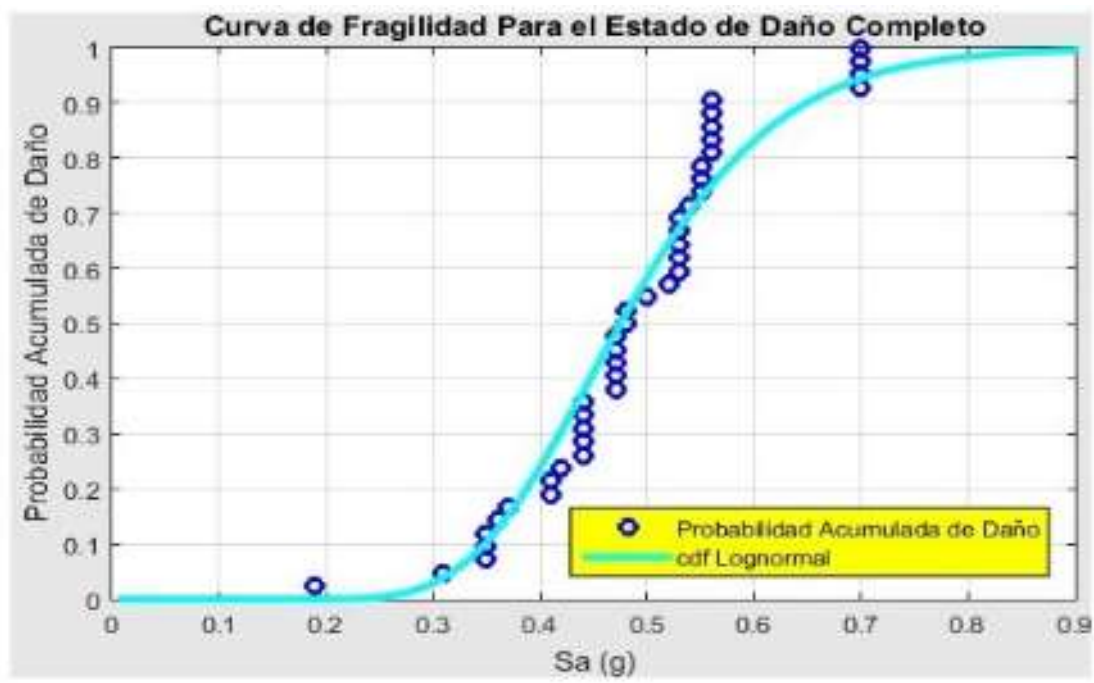

Fuente: Elaboración propia

Las curvas de fragilidad nos permiten saber el estado de daño de la edificación para cada evento sísmico, mediante una simulación aleatoria de la intensidad sísmica. En la siguiente ecuación el factor de daño (FD), representa la probabilidad de que el estado de daño ED sea mayor o igual al estado EDi.

$\mathrm{F} \mathrm{D}=(\mathrm{ED} \geq \mathrm{EDi} / \mathrm{ISi}) ;$ donde, $\mathrm{ISi}=$ Sagi $(5)$

Si representamos los estados de daño en una sola figura ya que todos los registros de la tabla $\{$ datosleve $\},\{$ datos moderado\}, \{datossevero $\},\{$ datoscompleto $\}$, guardan un relación entre si, según la intensidad sísmica se tiene:

Figura 11: Curva de fragilidad y estado de daño para $a(g)=0 ; 30$.

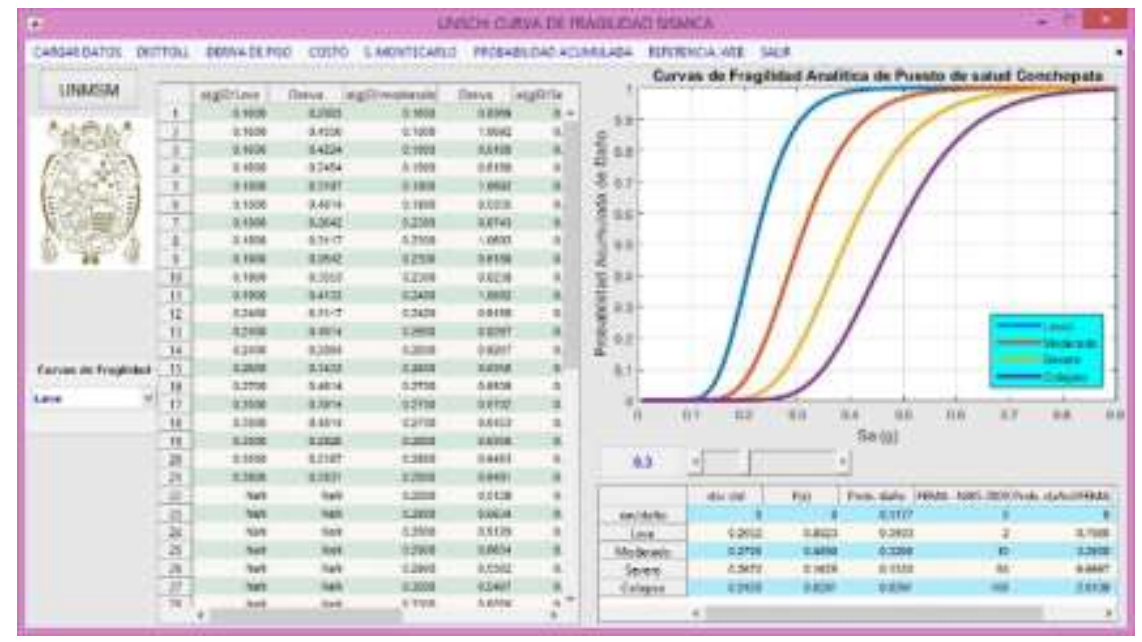

Fuente: Elaboración propia 
Se puede notar que la función de probabilidad acumulada se ajusta bastante a una función de distribución log normal por lo que para tener una mayor exactitud en los resultados se analizara en la sección siguiente las curvas de fragilidad haciendo una Simulación de Montecarlo para n variables.

\subsection{Montecarlo}

Para realizar la Simulación de Montecarlo es necesario trabajar con la probabilidad de ocurrencia de cada evento sísmico que produce un cierto nivel de daño, el cual se obtiene con la clasificación del número total de eventos sísmicos que producen un determinado estado de daño es decir se obtuvo la frecuencia según la intensidad sísmica, y de esta manera se calcula la probabilidad de ocurrencia de cada evento sísmico. En las figuras $(7,8,9,10)$, se muestran cada registro sísmico en función de la frecuencia y probabilidad de ocurrencia, el método de Simulación de Montecarlo para generar variables aleatorias, que nos permita obtener la función de probabilidad más fiable en la determinación de la probabilidad de ocurrencia, por el cual se generará números aleatorios probables relacionado a cada nivel de daño según el cuadro siguiente y de acuerdo a la figura11:

Cuadro 11: Probabilidad de ocurrencia para la Simulación de Montecarlo.

\begin{tabular}{|c|c|c|c|c|c|c|c|c|c|c|c|}
\hline \multicolumn{3}{|c|}{$\begin{array}{l}\text { Estado de daffo } \\
\text { leve }\end{array}$} & \multicolumn{3}{|c|}{$\begin{array}{l}\text { Estado de daño } \\
\text { moder ado }\end{array}$} & \multicolumn{3}{|c|}{$\begin{array}{c}\text { Estado de daño } \\
\text { colapso }\end{array}$} & \multicolumn{3}{|c|}{$\begin{array}{c}\text { Estado de daño } \\
\text { comple to }\end{array}$} \\
\hline Sismo & $\begin{array}{l}\text { Nro } \\
\text { Reg. }\end{array}$ & $\begin{array}{c}\text { Prob. } \\
\text { de } \\
\text { ocurren } \\
\text { cla }\end{array}$ & Sismo & $\begin{array}{l}\text { Nro } \\
\text { Reg }\end{array}$ & $\begin{array}{c}\text { Prob. } \\
\text { de } \\
\text { ocurren } \\
\text { cia }\end{array}$ & Sismo & $\begin{array}{l}\text { Nro } \\
\text { Reg. }\end{array}$ & $\begin{array}{c}\text { Prob. de } \\
\text { ocurrenc } \\
\text { ia }\end{array}$ & Sismo & $\begin{array}{r}\text { Nro } \\
\text { Reg. }\end{array}$ & $\begin{array}{c}\text { Prob. } \\
\text { de } \\
\text { ocurron } \\
\text { cia }\end{array}$ \\
\hline $0.16 \mathrm{~g}$ & 4 & 0.19 & 0.16 & 1 & 0.02 & $0,19 \mathrm{a}$ & 1 & 0.02 & $0.19 \mathrm{~g}$ & 1 & 0.02 \\
\hline $0.18 \mathrm{~g}$ & 4 & 0.19 & $0.18 \mathrm{~g}$ & 1 & 0.02 & $0.23 \mathrm{~g}$ & 1 & 0.02 & $0.31 \mathrm{~g}$ & 1 & 0.02 \\
\hline $0.19 \mathrm{~g}$ & 3 & 0.14 & $0.19 \mathrm{~g}$ & 4 & 0.08 & $0.26 \mathrm{~g}$ & 1 & 0.02 & $0.35 \mathrm{~g}$ & 3 & 0.07 \\
\hline $0.24 \mathrm{~g}$ & 3 & 0.14 & $0.23 \mathrm{~g}$ & 4 & 0.08 & $0.27 \mathrm{~g}$ & 1 & 0.02 & $0.36 \mathrm{~g}$ & 1 & 0.02 \\
\hline 0.260 & 1 & 0.05 & $0.24 \mathrm{~g}$ & 2 & 0.04 & $0.28 \mathrm{~g}$ & 1 & 0.02 & $0.37 \mathrm{~g}$ & 1 & 0.02 \\
\hline $0.27 \mathrm{~g}$ & 1 & 0.05 & $0.26 \mathrm{~g}$ & 3 & 0.06 & $0.29 \mathrm{~g}$ & 1 & 0.02 & $0.41 \mathrm{~g}$ & 2 & 0.05 \\
\hline $0.30 \mathrm{~g}$ & 4 & 0.19 & $0.27 \mathrm{~g}$ & 3 & 0.06 & $0.31 \mathrm{~g}$ & 4 & 0.09 & $0.42 \mathrm{~g}$ & 1 & 0.02 \\
\hline $0.38 \mathrm{~g}$ & 1 & 0.05 & $0.28 \mathrm{~g}$ & 4 & 0.08 & $0.33 \mathrm{~g}$ & 1 & 0.02 & $0.44 \mathrm{~g}$ & 5 & 0.12 \\
\hline \multirow[t]{15}{*}{ total } & 21 & 1 & $0.29 \mathrm{~g}$ & 4 & 0.08 & $0.34 \mathrm{~g}$ & 1 & 0.02 & $0.47 \mathrm{~g}$ & 5 & 0.12 \\
\hline & & & $0.30 \mathrm{~g}$ & 1 & 0.02 & $0.35 \mathrm{~g}$ & 2 & 0.05 & $0.48 \mathrm{~g}$ & 2 & 0.05 \\
\hline & & & $0.33 \mathrm{~g}$ & 4 & 0.08 & $0.36 \mathrm{~g}$ & 2 & 0.05 & $0.50 \mathrm{~g}$ & 1 & 0.02 \\
\hline & & & $0.34 \mathrm{~g}$ & 4 & 0.08 & $0.37 \mathrm{~g}$ & 5 & 0.12 & $0.52 \mathrm{~g}$ & 1 & 0.02 \\
\hline & & & $0.36 \mathrm{~g}$ & 2 & 0.04 & $0.38 \mathrm{~g}$ & 1 & 0.02 & $0.53 \mathrm{~g}$ & 5 & 0.12 \\
\hline & & & $0.37 \mathrm{~g}$ & 4 & 0.08 & $0.39 \mathrm{~g}$ & 1 & 0.02 & $0.54 \mathrm{~g}$ & 1 & 0.02 \\
\hline & & & $0.38 \mathrm{~g}$ & 3 & 0.06 & $0.41 \mathrm{~g}$ & 3 & 0.07 & $0.55 \mathrm{~g}$ & 3 & 0.07 \\
\hline & & & $0.39 \mathrm{~g}$ & 4 & 0.08 & $0,42 \mathrm{~g}$ & 3 & 0.07 & $0.56 \mathrm{~g}$ & 5 & 0.12 \\
\hline & & & $0.42 \mathrm{~g}$ & 1 & 0.02 & $0,48 \mathrm{~g}$ & 3 & 0.07 & $0.70 \mathrm{~g}$ & 4 & 0.10 \\
\hline & & & $0.50 \mathrm{~g}$ & 2 & 0.04 & $0.50 \mathrm{~g}$ & 2 & 0.05 & total & 42 & 1.00 \\
\hline & & & $0.54 \mathrm{~g}$ & 1 & 0.02 & $0.52 \mathrm{~g}$ & 4 & 0.09 & & & \\
\hline & & & total & 52 & 1 & $0.54 \mathrm{~g}$ & 2 & 0.05 & & & \\
\hline & & & & & & $0.55 \mathrm{~g}$ & 2 & 0.05 & & & \\
\hline & & & & & & $0,70 \mathrm{~g}$ & 1 & 0.02 & & & \\
\hline & & & & & & total & 43 & 1 & & & \\
\hline
\end{tabular}

Fuente: Elaboración propia 
Se utiliza el teorema del rechazo, es decir si el número aleatorio es mayor a la probabilidad de ocurrencia del sismo el evento se anula, pero si el número aleatorio es menor significa que si existe probabilidad de que suceda. Se realiza la simulación de Montecarlo para "n" números aleatorio generados para cada estado de daño, es decir mientras mayor es el valor de "n", la combinación de todos los resultados posibles es más exacto.

De todos los números aleatorios obtenidos se encuentra la media y la desviación estándar, para encontrar la función de probabilidad. A partir de la media y desviación estándar se integra a la función de distribución log normal el cual varía según el valor de " $\mathrm{n}$ " tomado. En la figura siguiente se presenta el diagrama de flujo, para la realización del programa en Matlab, donde el valor de prob_suc representa el valor obtenido del cuadro.

Figura 12: Diagrama de flujo para la Simulación de Montecarlo

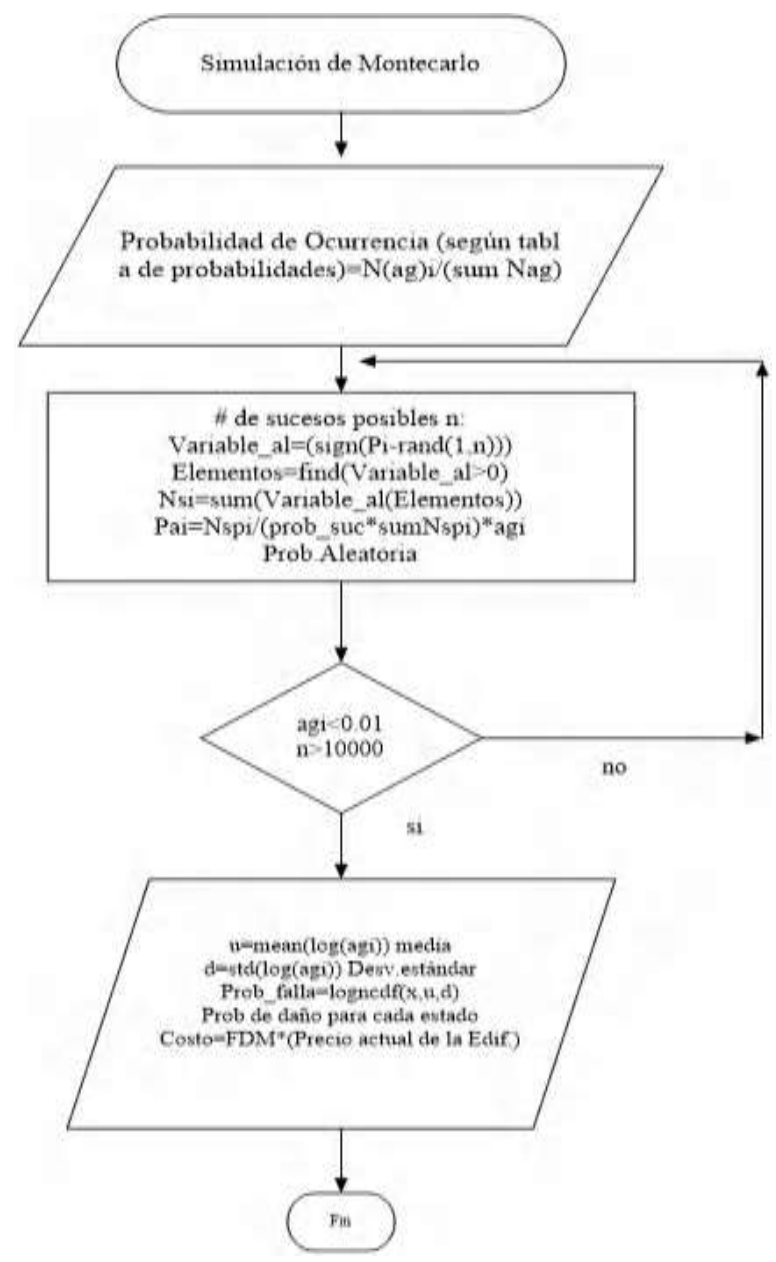

Fuente: Elaboración propia 
Figura 13: Simulación de Montecarlo G.U.I

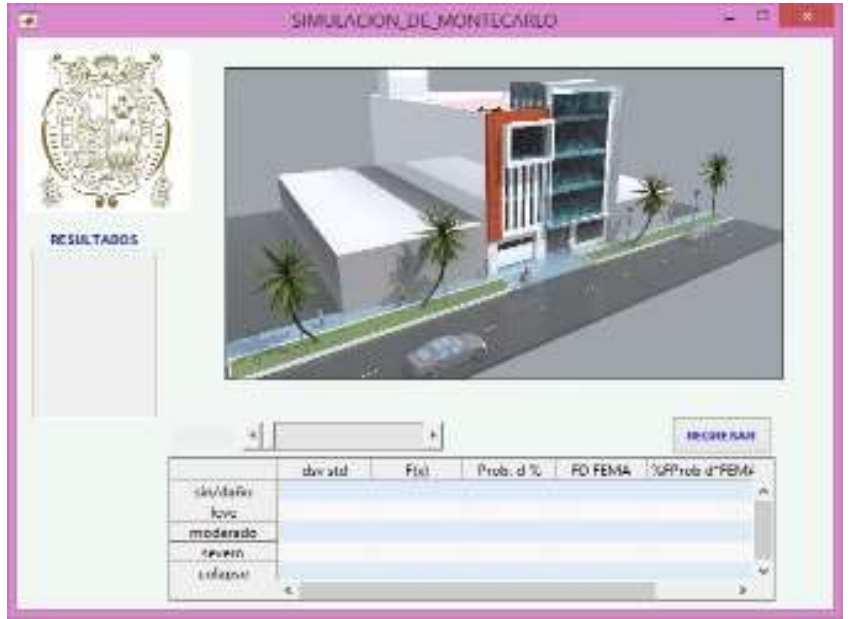

Fuente: Elaboración propia

Figura 14: Simulación de Montecarlo, $a(g)=0 ; 20$.

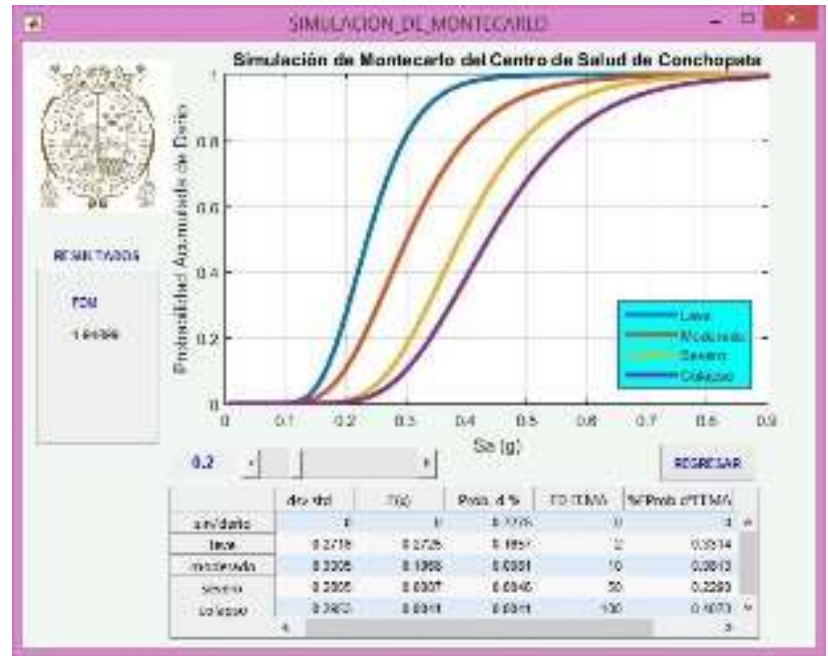

Fuente: Elaboración propia

Figura 15: Simulación de Montecarlo, $a(g)=0 ; 30$.

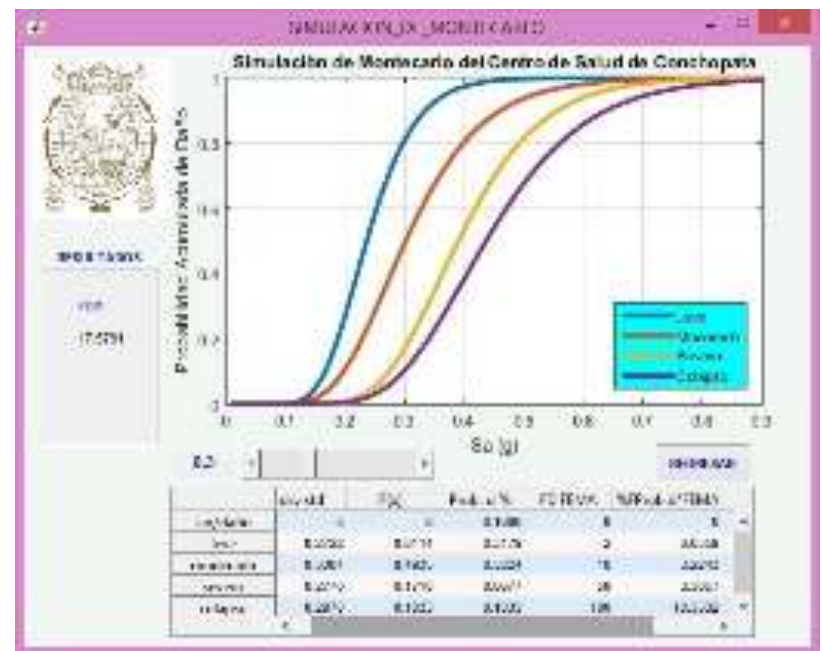

Fuente: Elaboración propia 
Figura 16: Simulación de Montecarlo, $a(g)=0 ; 40$.

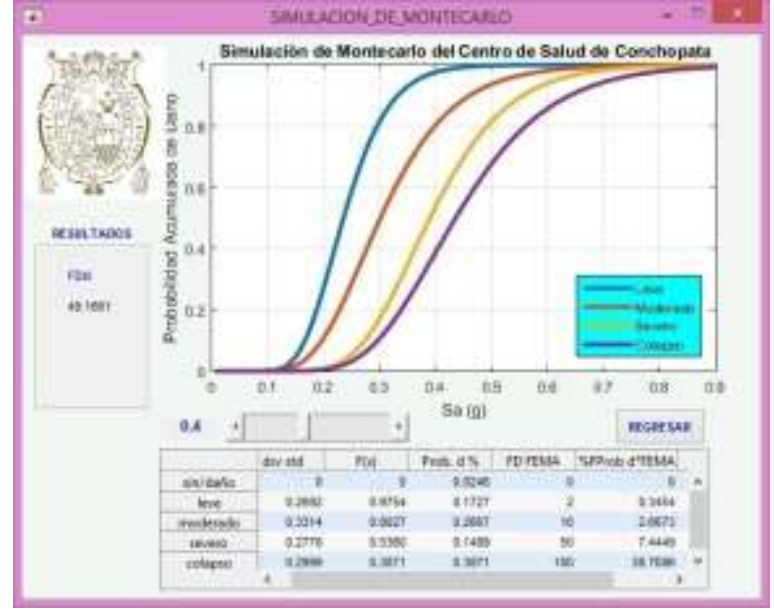

Fuente: Elaboración propia

\section{RESULTADOS}

\subsection{De las C.F.A con Intensidades sísmicas}

De la investigación realizada de peligro sísmico en el Departamento de Ayacucho, para un periodo de retorno de 50 años y probabilidad de excedencia de $10 \%$, se estima una intensidad sísmica menor e igual a $0.32 \mathrm{~g}$, por el cual a continuación se presenta los resultados de las pérdidas económicas para diferentes intensidades de peligro sísmico, analizado con el método de Simulación de Montecarlo. Representación de las curvas de fragilidad para una intensidad sísmica de $0.20 \mathrm{~g}$, para cada estado de daño, en función de la probabilidad de excedencia figura18.

Figura 17: G.U.I de la Simulación de Montecarlo

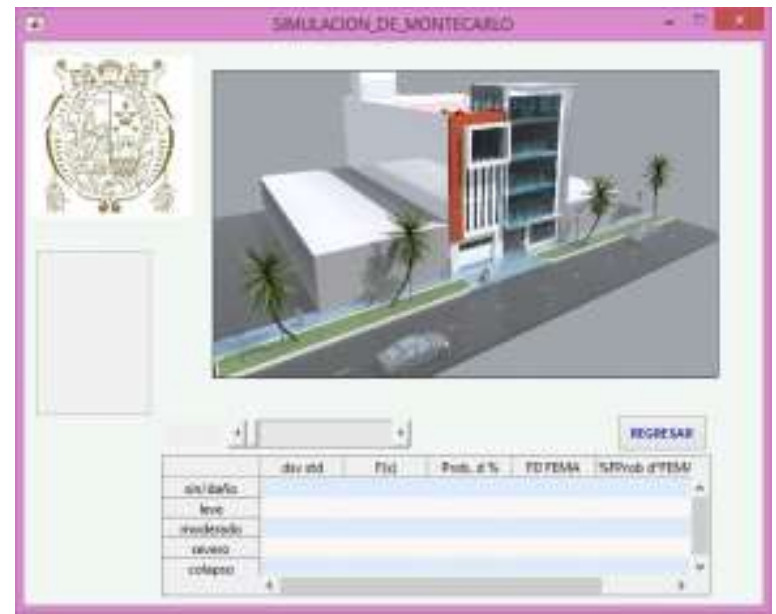

Fuente: Elaboración propia 
Valenzuela...

Figura 18: Curvas de fragilidad para una intensidad sísmica, $a(g)=0 ; 20$

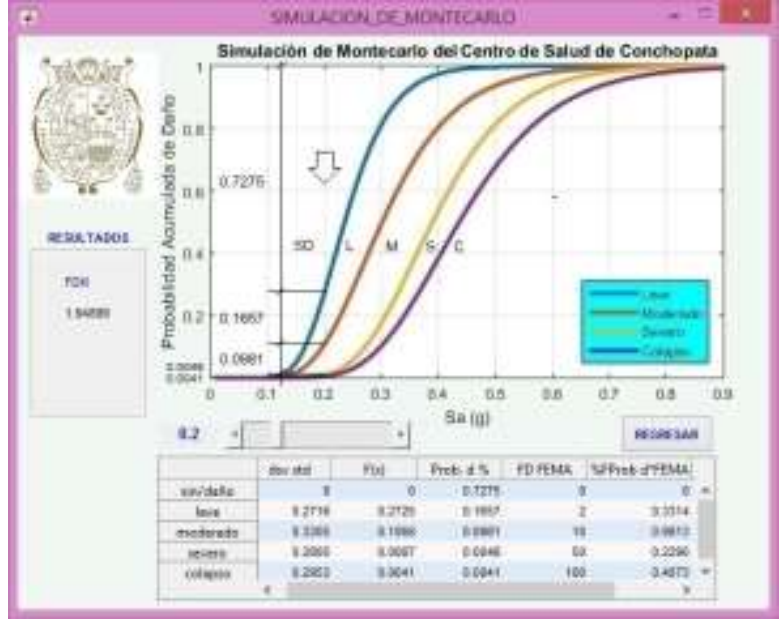

Fuente: Elaboración propia

Figura 19: Curvas de fragilidad para una intensidad sísmica, $a(g)=0 ; 30$

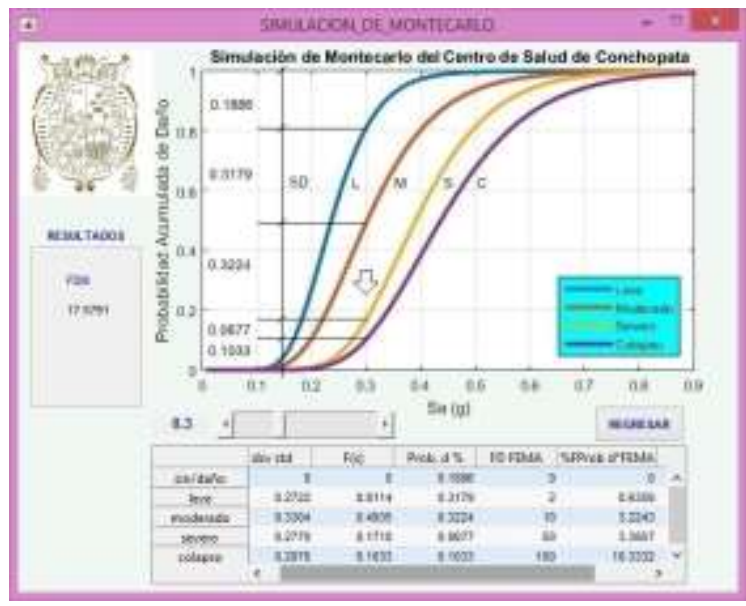

Fuente: Elaboración propia

Figura 20: Curvas de fragilidad para una intensidad sísmica, $a(g)=0 ; 40$

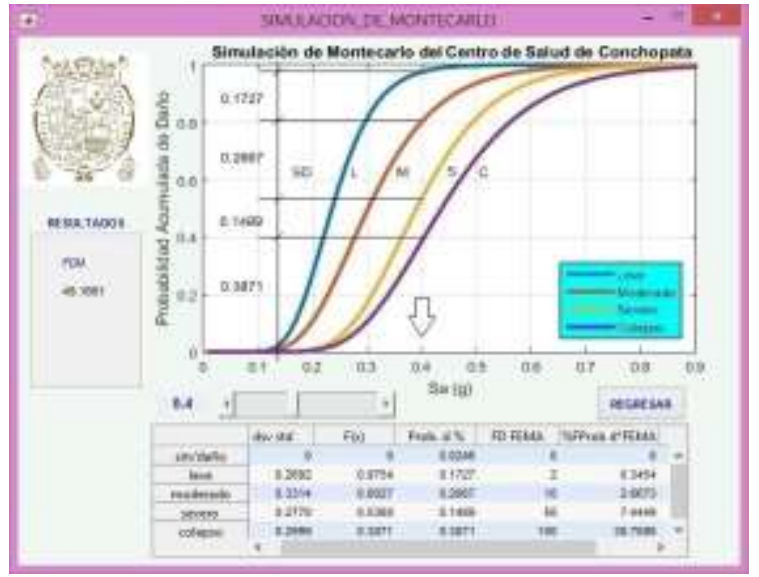

Fuente: Elaboración propia 


\subsection{De las C.F.A con Deriva de piso}

Con la metodología empleada para obtener las curvas de fragilidad con respecto a la intensidad sísmica se obtuvo resultados teniendo como parámetro fundamental la deriva de piso, clasificado según el cuadro1 propuesto por Ghobarah. En la figura22 se presenta la curva de fragilidad en función de la deriva de piso obtenido en $\%$, el cual permite diferenciar el daño estructural de la edificación ante una determinada distorsión de piso, como también permite saber el nivel de daño estructural para la deriva propuesta por la norma E030 que es de $0.7 \%$.

Figura 21: G.U.I de Curvas de fragilidad según la deriva de piso

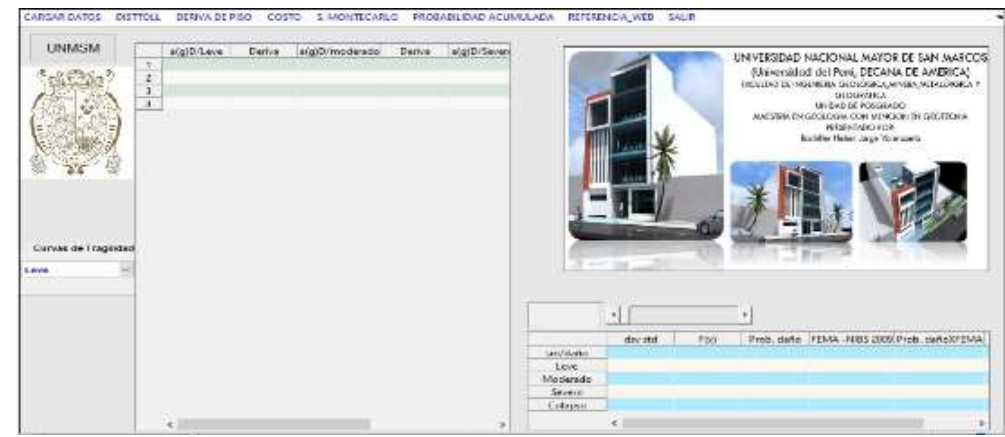

Fuente: Elaboración propia

Figura 22: Ingreso a las derivas de piso G.U.I Curvas de fragilidad

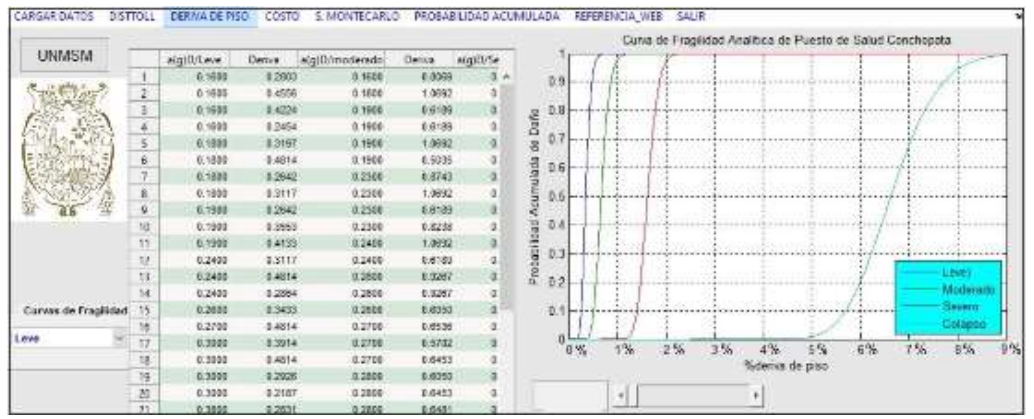

Fuente: Elaboración propia

Figura 23: Curvas de fragilidad ampliado según las derivas de piso

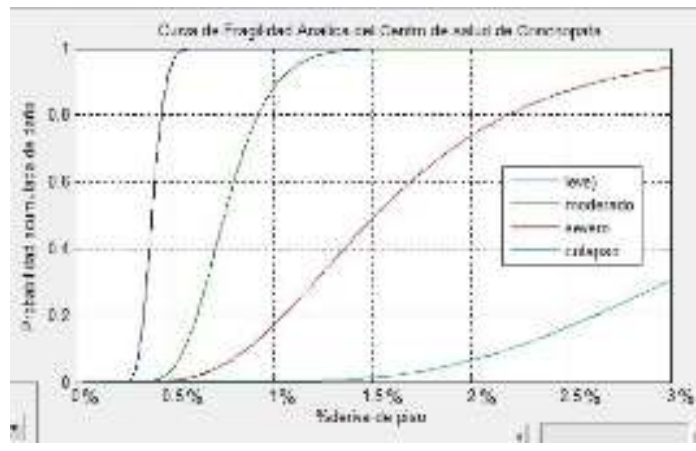

Fuente: Elaboración propia 
Figura 24: Curvas de fragilidad ampliado según las derivas de piso

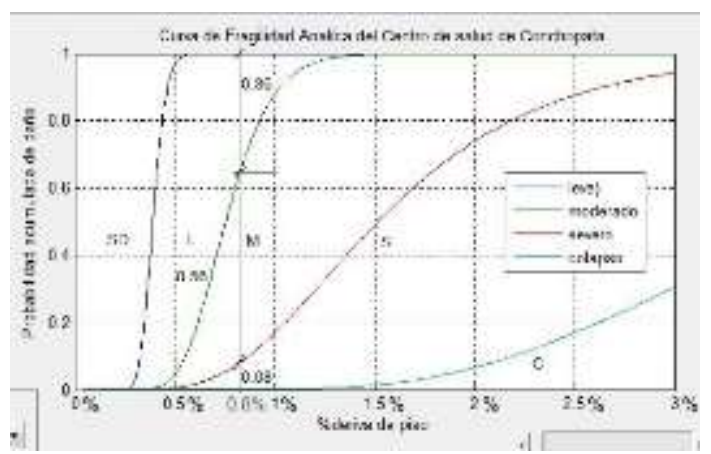

Fuente: Elaboración propia

\section{DISCUSIÓN}

Con los resultados obtenidos en las Curvas de Fragilidad Analítica según las intensidades sísmicas de los 30 registros aplicadas en la base de la estructura se pudo comprobar que existe una función de distribución acumulada, que nos arroja resultados de la más probable y mediante una correlación con la cuadro 1 de Ghobarah (Deriva vs Daño) (Ghobarah (1997)) y tomando como referencia la metodología de factores de daño por el modelo HAZUS (FEMA \& NIBS, 2009) cuadro 5, se obtiene la descripción completa del tipo de daño generado en la estructura. Es preciso aclarar que el Método de la simulación de Montecarlo, se usa para diferentes simulaciones aleatorias, mientras mayor sea el valor de " $n$ " asumido para la generación de variables aleatorias, la determinación del tipo de daños encontrado del Edificio para una intensidad sísmica es más óptima.

\section{Análisis de la Curva de Fragilidad según a $(\mathrm{g})=0.20 \mathrm{~g}$}

Para una intensidad de $0.20 \mathrm{~g}$ y una simulación aleatoria de Montecarlo de 158 puntos se obtiene un valor de 0.7275 (72.75\%), de que la estructura después de un sismo quede sin daño o presente grietas menores localizadas.

Presenta un daño leve de 0.1657 (16.57\%), quiere decir que la estructura presentara grietas ligeras en toda la estructura. Presenta un daño moderado de 0.0981 (9.81\%), lo que significa que la estructura presenta grietas severas y desprendimiento localizados. Presenta un daño severo de 0.041 (4.1\%), es un valor muy pequeño, pero existe aplastamiento del concreto armado, refuerzo visible. Presenta un daño severo de 0.0046 $(0.46 \%)$, es un valor muy pequeño

pero existe aplastamiento del concreto armado, refuerzo visible. Presenta una probabilidad de colapso de $0.00041(0.041 \%)$, es un valor muy pequeño, pero representa a que ante un sismo de $0.20 \mathrm{~g}$ la estructura pueda colapsar, es decir la probabilidad a que 
suceda esto es insignificante. El factor de daño total se obtiene del producto del factor de daño obtenido anteriormente con el Factor de daño de perdidas propuesto por HAZUS FEMA para edificaciones en general, lo cual para un sismo de $0.20 \mathrm{~g}$ obtenemos un FDM de $1.94899 \%$ (factor de daño medio).

\section{Análisis de la Curva de Fragilidad según $\mathrm{a}(\mathrm{g})=0.30 \mathrm{~g}$}

Con una simulación aleatoria de Montecarlo se obtiene una probabilidad de excedencia de 0.1886 (18.860) \% ( $\sin$ daño), significa que solo se presente grietas menores localizadas en la estructura. Existe una probabilidad de 0.3179 (31.79\%) de daño leve, quiere decir que la estructura presenta grietas ligeras en toda la estructura. Presenta un daño moderado de 0.3224 (32.24\%), lo que significa que la estructura presenta grietas severas y desprendimiento localizados. Presenta un daño severo de 0.0677 (6.77\%), existe aplastamiento del concreto armado, refuerzo visible. Presenta una probabilidad de colapso de $0.1033(10.33 \%)$, representa el porcentaje de que la estructura pueda colapsar. El factor de daño total se obtiene del producto del factor de daño obtenido anteriormente con el Factor de daño de pérdidas propuesto por HAZUS FEMA para edificaciones en general, lo cual para un sismo de $0.30 \mathrm{~g}$ obtenemos un factor de daño medio (FDM) de $17.5791 \%$.

\section{Análisis de la Curva de Fragilidad según a $(\mathrm{g})=0.40 \mathrm{~g}$}

Para este tipo de intensidad sísmica $(0.40 \mathrm{~g})$ no corresponde a la zona 2 de Ayacucho, pero según la simulación obtenido es bueno ver como es el comportamiento estructural ante este tipo de sismos. Existe una probabilidad de 0.246 (2.46 \%) de no presentar daño alguno. Existe una probabilidad de 0.1727 (17.27 \%) de daño leve. Presenta un daño moderado de 0.2667 (26.67\%).

Presenta un daño severo de 0.1489 (14.89\%). Presenta una probabilidad de colapso de 0.3871 (38.71\%). Obtenemos un factor de daño medio (FDM) de $49.1661 \%$. Resultados de las C.F.A según la deriva de piso de HAZUS-FEMA Según el cuadro1 de niveles de daño propuesto por (Ghobarah (1997), cuando la deriva es menor a $<0.2 \%$ no existe daño, pero cuando la deriva está en un rango de $0.20 \%<0.5 \%$, presenta un daño leve es decir presenta grietas ligeramente visibles. Cuando la deriva de piso esta entre los valores 0.5 $\%<1.1 \%$, se encuentra en un daño moderado, donde se observa grietas menores de $1 \mathrm{~mm}$ (fluencia del acero). 
Para distorsiones de piso entre $1.1 \%<2.3 \%$, la estructura se encuentra en daño extensivo o severo y presenta grietas de 1 a $2 \mathrm{~mm}$. Para distorsiones de piso mayores a $<2.3 \%$, la estructura se encuentra en daño completo es decir presenta grietas mayores $2 \mathrm{~mm}$.

\section{CONCLUSIONES}

- Después de realizar las evaluaciones correspondientes y utilizar los métodos ya expuestos anteriormente, se concluye que la determinación de curvas de fragilidad analítica a través del análisis incremental dinámico tiempo historia es un factor determinante para prevenir los estados de daños causados por el sismo.

- En el procedimiento de análisis incremental dinámico en el centro de salud de Conchopata en Huamanga-Ayacucho, permitió generar los estados de daño según las incursiones sísmicas generadas en la base del centro de salud de Conchopata en Huamanga-Ayacucho.

- En todo diseño estructural de una edificación se debe tomar muy en cuenta la estimación de los efectos de sitio, la amplificación del movimiento sísmico esperada dadas las condiciones geológicas superficiales en el sitio de interés.

- La estimación de los efectos de sitio mejora enormemente el comportamiento sísmico de los diseños estructurales a la vez se obtiene diseños con fundaciones más óptimas.

- La respuesta del desplazamiento máximo de la estructura analizada se presenta cuando el movimiento sísmico alcanza una aceleración máxima esto ocurre con mayor frecuencia en el análisis incremental dinámico, por lo que en ese instante se registran las mayores solicitaciones sísmicas ocasionando diferentes niveles de daño en las zonas más vulnerable de la edificación.

- En un análisis dinámico tiempo historia se tiene que utilizar no menos de cinco registros de aceleraciones horizontales, correspondientes a sismos reales o artificiales, este registro sísmico tiene que ser normalizados y escalados, de esta manera nos aseguramos en tener respuestas más fiable y muy cercanas a la realidad.

- La estructura soporto deformaciones en sus componentes sin que se dañe gravemente o se degrade su resistencia, se concluye que diseños estructurales que tenga una buena rigidez lateral en ambas direcciones, resisten mejor las solicitaciones generadas por el sismo durante toda su vida útil de la edificación. Mientras mayor es el valor de "n" asumido para la generación de variables aleatorias, la determinación del tipo de daños 
encontrado del Edificio para una intensidad sísmica es más fiable, ya que se obtiene una distribución de probabilidad más óptima.

\section{AGRADECIMIENTOS}

A la facultad de Ingeniería Geológica, Minera, Metalúrgica y Geográfica de la Unidad de Posgrado de la Universidad Nacional Mayor de San Marcos, Universidad del Perú,

Decana De América que a través de sus docentes me brindó conocimientos y enseñanzas para el mejor desenvolvimiento en mi vida profesional. A mi alma Mater la "Universidad Nacional de San Cristóbal de Huamanga "por haberme brindado formación profesional.

\section{REFERENCIAS}

CISMID. (2013). Generación de acelerogramas sinteticos en convenio con sencico.

Obtenido de //www.sencico.gob.pe/gin/investigacion.html.

E030. (2018). E030 diseño Sismoresistente. Reglamento Nacional de Edificaciones.Diario el Peruano.

FEMA, \& NIBS. (2009). Earthquake Model,Technical Manual, Federal Emergency Management Agency (FEMA) and National Institute of Building Sciences(NIBS). Multi-hazard Loss Estimation Methodology.

Ghobarah. (1997). Criterio para la evaluación del desempeño estructural. Monograph series in Earthquake Engineering(CIMNE).

Velásquez, V. (2006). Estimación de Pérdidas por Sismo en Edificios peruanos mediante curvas de Fragiliada Analitica. Tsis PUCP, 134.

Francisco, M. (2013). Blog profesional de Francisco Martinez Alonso. Estructuras Sismorresistente Análisis modal espectral-XFMA.

García, B; González, S; Jornet, M. (2010). Material elaborado que fue adaptado del Tutorial SPSS 15. Grupo de Innovacion Educativa. Universidad de Valencia.

Garrido, R. (2015). Vulnerabilidad Sísmica en edificaciones porticadas compuestas de acero y hormigón armado.Tesis Doctoral. España: Universitat Politécnica de Catalunya.

Ghobarah. (1997). “Criterios para la evaluación del desempeño estructural. Monograph Series in Earthquake Engineering (CIMNE). Disponible en https: //www.scipedia.com/public/Aguiar_2006a. 
Ghobarah. (1997). Criterio para la evaluación del desempeño estructural. Monograph series in Earthquake Engineering(CIMNE).

Gil, S; L.Franco. (2005). Estimación de pérdidas por sismo en esdificios peruanos mediante curvas de fragilidad análiticas. IX Congreso Chileno de Sísmologia e Ingeniería antisísmica. Concepción, Chile.

Gomez, J. (2005). El Método de Montecarlo. Curso de Estadística TAE. Revisado en http://benasque.org.

Marín, G. (2012). Evaluación del riesgo sísmico del centro histórico de la Ciudad de Huánuco. Universidad Nacional de Ingeniería. Lima-Perú. Disponible en http: //cybertesis.uni.edu.pe/handle/uni/1236.

Palacios, L. (2004). Peligro Sísmico en el Dep. de Ayacucho. Tesis UNSCH.

Paz, E.K. (2012). Procedimiento de cálculo para la elaboración de espectros sísmicos para el diseño sismorresistente de estructuras. Trabajo de Graduación. Guatemala: Universidad de San Carlos de Guatemala Facultad de Ingeniería.

Sáenz, R. (2019). Vulnerabilidad sísmica en edificaciones esenciales mediante curvas de fragilidad analíticas en el edificio administrativo de la Universidad Nacional del Centro del Perú. Huancayo Perú. Tesis para optar el Titulo de Ingeniero Cívil. Perú: Facultad de Ingeniería Civil del Departamento de Estructuras.

Vargas, P; Barbat; Hurtado. (2013). Evaluación probabilista de la capacidad fragilidad y daño sísmico de edificios de hormigón armado. Revista Internacional de Métodos Numéricos para Cálculo y Diseño en Ingeniería Vol.29(2), 63-68 pp. Disponible en http://doi.org/10.1016/j.rimni.2013.04.003.

Velásquez, V. (2006). Estimación de Pérdidas por Sismo en Edificios Peruanos Mediante Curvas de Fragilidad Analíticas. Tesis Posgrado-PUCP, Lima Perú, 134p.

Zelaya, V.A. (2007). Estudio sobre Diseño Sísmico en construcciones de Adobe y su Incidencia en la Reducción de Desastres. Tesis de Maestria. Perú: Universidad Nacional Federico Villareal. Escuela Universitaria de Post Grado, Programa de Maestría en Gerencia de la Construcción moderna. 
Determinación de las curvas de fragilidad... 